\title{
Reproductive isolation from incompatible Hybrid Mitotic Exit Networks (HyMEN)
}

\author{
Pierre Hutter \\ Former Head of Medical Genetics, ICH-HVS, Sion, Switzerland. Co-founder \& former CSO, SOPHiA Genetics. \\ Address for correspondence: phutter@sophiagenetics.com
}

Key words: reproductive isolation, MEN, Hmr, Rab GTPases, cholesterol, HyMEN

\begin{abstract}
At the end of mitosis the Mitotic Exit Network (MEN) pathway triggers complex tasks which mainly include the spindle disassembly and the nuclear envelopes assembly. In the course of telophase, which often lasts less than an hour and corresponds to only about $2 \%$ of the entire cell cycle's duration, spatial and temporal cues are integrated to ensure that cytokinesis occurs after the genome has partitioned between mother and daughter cells. From the end of anaphase through telophase, sequential components of a Ras-like GTPase signaling pathway are controlled by a set of different spatial and temporal signals. Successful propagation of these signals through multi-step transduction requires a remarkable sequential coordination. By considering that cells lacking proper MEN function fail to exit from mitosis, I argue that in a hybrid genome impaired coordination between two diverged MENs is prone to result in critical mitotic defects, from late anaphase through telophase. The socalled HyMEN model of hybrid incompatibility depicted here can be regarded as an extension of the BatesonDobzhansky-Muller model of speciation, centered on the MEN.
\end{abstract}

\section{Evidence supporting the HyMEN model of reproductive isolation}

Figure 1 sketches an ancestral population that has split into two derived populations physically isolated for a substantial period of time. In the first derived population, mutation $A$ to a occurs in a gene comprised within a subset of MEN related genes (BC-D...), clustered around the major speciation gene $H m r^{1}$. The a mutation results in a structural change of a protein which first remains functionally neutral because it does not reach a different destination (or act with a different timing) compared to A. Nevertheless, in some individuals from subsequent generations carrying the a mutant, additional mutations occur in the linked genes B-C-D... (mutating to B'-C'-D'...), allowing for the altered protein encoded by a to achieve a novel function in telophase.

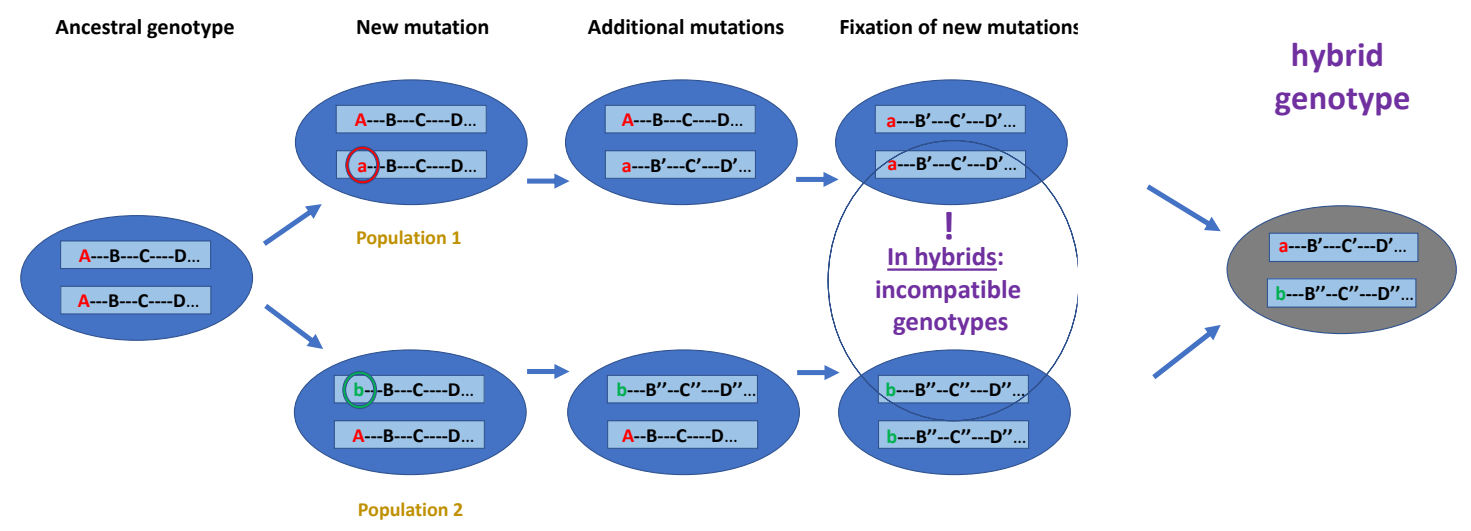

Figure 1. The HyMEN model of reproductive isolation. By pertaining to the Mitotic Exit Network, cumulative mutations in a set of genes clustered around $\mathrm{Hmr}$ result in hybrid incompatibilities.

The genetic variants implied in the proposed model are expected to mainly affect vesicles, cargos and motor proteins that control endocytosis related transport and attachment of the novel protein (encoded by gene a) to a different destination site, e.g. on a microtubule or a microfilament. Whenever the new set of mutated genes confers a selective advantage in the environment colonized by the first derived population, positive selection on the haplotype a-B'-C'-D' is expected to build up and stabilize a syntenic block which maximizes the spatial, temporal and directional regulation of the novel a protein. If positive selection on $a-B^{\prime}-C^{\prime}-D^{\prime}$... genes is sustainable (for instance by allowing their carriers to make a better use of cholesterol available in a newly identified source of food), the derived haplotype would become fixed, as homozygous genotype, in this first population. Similarly, in a second population derived from the same ancestral population, a distinct mutation of $A$ 
to $b$ and its subsequent changes in genes B-C-D... (mutating to B'- - C'-D'... ) lead to the fixation of $a$ different syntenic block (b-B' '-C''-D'...). Once the two derived populations have gone through sufficient genetic divergence at the same set of clustered genes, a hybrid genotype composed of the juxtaposition of $a-B^{\prime}-C$ '-D'... and $b-B$ ' '-C' '-D'... haplotypes is likely to exhibit light to severe genetic incompatibilities manifested as hybrid breakdown, hybrid sterility or hybrid inviability, thus bringing about post-mating reproductive isolation.

As previously suggested in the "Rabed Queen Hypothesis" (P. Hutter, Flybase 2009), exogenous cholesterol could be a key environmental trigger to promote the evolution of divergent clusters of MEN relevant genes encompassing $\mathrm{Hmr}$, including its 6 surrounding paralogous Rab GTPases which regulate endocytosis through decorating and targeting intracellular vesicles to appropriate subcellular compartments. Remarkably, in D. melanogaster these 7 particularly fast evolving genes have remained tightly linked for at least $45 \mathrm{Myr}$ in the middle of the $X$ chromosome, where they have been subject to very strong positive selection ${ }^{2}$. RAB GTPases substrates are activated and consequently stabilized on membranes by guanine nucleotide exchange factors (GEFs). These are the primary determinants of localization of GTP bound RAB GTPases which, once activated by their specific GEF, recruit their downstream effectors that mediate vesicular transport sub-steps. For instance, the TRAPP (transport protein particle) II complex acts as a GEF at least for the Golgi gatekeepers RAB1, 31 and $32^{3}$. Remarkably, the 6 Rab GTPases paralogs ${ }^{2,4}$ clustered around $\mathrm{Hmr}$ are perdicted to interact in lipid homeostasis with the TRAPPII complex, which binds to a component of the COPI complex required for limiting lipid storage. The interaction between COPI and TRAPPII may bring TRAPPII onto the surface of lipid droplets, and at least RABX2 (encoded by one of the above 6 Rab GTPases paralogs) is involved in lipid droplet organization where it interacts with the OSBP protein that controls membrane cholesterol (see Discussion). As an essential component of all animal cell plasma membranes, cholesterol is needed at the end of mitosis for the synthesis of new phospholipid bilayer membranes. Thus, a subset of MEN relevant genes clustered around $\mathrm{Hmr}$ in the middle of the X chromosome, and including Hmr's 6 linked Rab GTPases paralogs, members of the TRAPPII/COPI complexes, as well as the Ran GTPase (regulating cholesterol biosynthesis) that lies only $192 \mathrm{~kb}$ away from the Rab9Fb paralog, are expected to collaborate in a highly orchestrated process during the short period of telophase. Thus, at the end of mitosis interactions between Rab GTPases likely involved in clathrin-mediated endocytosis, and genes associated with the TRAPPII/COP1 complexes, stand out as major players in lipogenesis and lipolysis, to ensure efficient vesicle traffic with docking proteins at their destination site ${ }^{5}$.

Part of the multi-subunit TRAPPII tethering complex is composed of BET3, BET5, TRS20, TRS23, TRS31, TRS33 and TRS 85 proteins. The complex, by binding to a component of the COPI coat, provides the initial recognition event that links a particular vesicle with its target membrane, participating in intra-Golgi and endosome-Golgi transport. With regard to this it is worth noting that BET3, BET5, TRS23 and TRS31 are predicted to interact with RAB9D (encoded by the paralog Rab9D which lies only 950 bp away from $\mathrm{Hmr}$ in D. melanogaster and even less in most other Drosophila species).

By considering that bringing the initial $A$ allele to functional deployment of mutants $a$ or $b$ depends on complementary changes in the postulated subset of genes pertaining to the MEN, the present model suggests that reproductive isolation may often result from incompatibility between diverged MEN related genes in hybrids. Moreover, it should be emphasized that the presence in $D$. melanogaster of the same 359 bp repeat satellite flanking at least 8 genes $^{2}$ of the MEN related cluster, points to the possibility of a direct surveillance of these repeats by $\mathrm{Hmr}$, through its role of a major repressor of overall transposition. The present hypothesis is largely based on genetic analyses published elsewhere ${ }^{2,4}$ and communications addressed to the Flybase (https://flybase.org/).

Multiple roles of MEN related genes at telophase During the relatively short duration of telophase, highly coordinated MEN genes not only build up two nuclear envelopes (NEs) but also work as architects of the interphase chromatin structure. Indeed, the NE defines an essential structure for cellular organization as it is responsible for the separation of nucleoplasm from cytoplasm and the respective biological processes including transcription and translation that operate therein. As nuclear membranes start to reassociate with chromatin during the late stages of anaphase, most robust temporal and spatial coordination is required between chromatin and nuclear membranes dynamics. Just as various proteins must stabilize the membrane curvature at these sites, mechanisms must restrict the diffusion of membrane lipids between the endoplasmic reticulum (ER), the nascent outer nuclear membrane and the nascent inner nuclear membrane $\left(\right.$ INM) ${ }^{6}$. The INM is an adaptable membrane territory active in lipid metabolism, and cells can target enzymes to the INM to promote lipid storage ${ }^{7}$. As highly dynamic organelles involved in the regulation of both intracellular lipid storage and lipid metabolism, lipid droplets are essential players in the above process. Besides, the association of INM proteins with chromatin is largely regulated by retrograde transport receptors and by the already mentioned RAN GTPase. Noteworthy, the relative abundance of ER sheets and tubules needs to be finely tuned since it varies between mitotic cell types, organs and differentiation states. At the destination of this transport, the organization of membranes is mediated by lipids that help membrane vesicles bind chromatin to give rise to the NE. Furthermore, distinct lipid compositions contribute to functional partitioning at the NE, whose vesicles are specifically enriched in phosphoinositides, which confer a specific level of fluidity at the membrane ${ }^{8}$. Indeed, each cellular membrane compartment uses a characteristic species of phosphoinositide, whose signature attracts a specific complement of loosely 
attached peripheral proteins to that membrane. Since their activity decreases when the abundance of this lipid falls, phosphoinositides contribute to the selection of peripheral proteins for each membrane and regulate the activity of the integral proteins. Thus, the regulated recruitment of membrane proteins of the INM mediates the accumulation of nuclear envelope-specific membranes as well as the establishment of a distinct ER subdomain.

\section{Discussion}

At the end of mitosis membrane-attached GTPbound RABs along with their downstream effectors which include all the components of vesicular transport machinery are collectively required for vesicle formation, motility, tethering, and fusion. As emphasized above, the OSBP protein involved in lipid droplet organization interacts with RABX2 (encoded by one of the 6 Rab GTPases paralogs clustered around $\mathrm{Hmr}$ ). Both the endosomal cholesterol trafficking defective npc1 mutants and the non-vesicular mediated intracellular sterols trafficking defective Osbp mutants exhibit similar failure in Drosophila spermatogenesis. More precisely, npc1 mutants show abnormal sterol accumulation in aberrant multi-lamellar body-like

\section{References}

1. Hutter, P., and Ashburner M. Genetic rescue of inviable hybrids between Drosophila melanogaster and its sibling species. Nature 327, 331-333 (1987).

2. Hutter, P. Rapidly evolving Rab GTPase Paralogs and Reproductive Isolation in Drosophila. Advances in Genetics, Vol. 58, 1-23 (2007).

3. Lipatova, Z. and Segev, N. Ypt/Rab GTPases and their TRAPP GEFs at the Golgi. FEBS Letters, 593, 2488-2500 (2019).

4. Hutter, P. X-linked small GTPase and OXPHOS genes are candidates for the genetic basis of hybrid inviability in Drosophila. Dev. Genes Evol. 212, 504-512 (2002). organelles. With regard to this it is worth noting that all 6 Rab GTPases paralogs clustered around $\mathrm{Hmr}$ are mostly expressed in testis. Mutants of FAN, a VAMPassociated endoplasmic reticulum protein which interacts with OSBP, also have a defect in sterol distribution and individualization.

For their movement between different compartments within the cell, vesicles rely on the motor proteins myosin, kinesin (primarily anterograde transport) and dynein (primarily retrograde transport). One end of these motor proteins attaches to the vesicle while the other end attaches to either microtubules or microfilaments 9 . The motor proteins then move by hydrolyzing ATP, which propels the vesicle towards its destination. As a vesicle nears its intended location, RAB GTPases proteins in the vesicle membrane are the key interactors with docking proteins at the target site. When considering that reproductive isolation may be a consequence of incompatibility between diverged HyMEN related genes, specific tissues may be associated with more critical mitoses than others. Indeed, since different contributions from the MEN are expected in various mitotic cell types and differentiation states, it is possible that particularly sensitive steps in specific telophases serve as checkpoints assessing the performance and robustness of mitosis.

5. Angers, C.G. and Merz A.J. New links between vesicle coats and Rab-mediated vesicle targeting. Semin Cell Dev Biol, 22, 18-26 (2010).

6. Bahmanyar S. and Schlieker C. Lipid and protein dynamics that shape nuclear envelope identity. Mol Biol Cell 31, 1315-1323 (2020).

7. Romanauska A. and Köhler A. The inner nuclear membrane is a metabolically active territory that generates nuclear lipid droplets. Cell 174, 700-715 (2018). 8. Jackson C.L., Walch L and Verbavatz J-M. Lipids and their trafficking: an integral part of cellular organization. Developmental Cell 39,139-153 (2016).

9. Sweeney H.L. and Holzbaur E.L.F. Motor Proteins. Cold Spring Harbor Perspectives in Biology (2021). 